\title{
APLICAÇÃO DE METODOLOGIA PROBLEM-BASED LEARNING A PARTIR DO PONTO DE VISTA DO PROFESSOR
}

\author{
Application of problem-based learning methodology from the teacher's point of view
}

Tiago Rafael Sausen - UNIVEL/Brasil

\begin{abstract}
RESUMO: O presente artigo apresenta as reflexões de um docente no desenvolvimento da metodologia de Aprendizagem Baseada em Problemas (PBL - Problem Based Learning) em uma disciplina de Psicofarmacologia. O PBL caracteriza-se por apresentar um problema que não apresenta uma solução pré-definida e não se foca muito na resolução do problema em si, mas sim no empenho de propor soluções. A partir dos registros das ações realizadas e de um processo de reflexão do resultado delas, foi possível ao docente avaliar o processo de implementação de uma metodologia ativa de aprendizagem. A principal característica da aplicação do PBLé a necessidade de mudança de mentalidade e comportamento do professor, pois o mesmo precisa criar um ambiente onde o processo de aprendizagem passa a ser centrado no estudante, e o docente precisa instigá-lo a aprender, demonstrando a importância e desenvolvendo sua autonomia. A utilização de metodologias que aproximem o estudante das situações que ele encontrará na sua vida profissional garante um maior engajamento deles nas aulas. $\mathrm{O}$ estudo aqui apresentado evidenciou a importância da inclusão de metodologias ativa em salas de aula, pois a capacidade dos estudantes aprenderem é melhor desenvolvida. Entretanto, como não há uma fórmula pronta para uso dessas metodologias, o entendimento, a escolha e o desenvolvimento de metodologias ativas de aprendizagem exigem uma grande dedicação do professor durante todo o processo de ensino.
\end{abstract}

PALAVRAS-CHAVE: Educação. Metodologia ativa. Psicologia.

ABSTRACT: This work presents the teacher's reflections in the application of the Problem Based Learning (PBL) methodology in a Psychopharmacology discipline. The PBL is characterized by presenting a problem that does not have a predefined solution and does not focus much on solving the problem itself, but on the commitment to propose solutions. From the records of the actions carried out and a reflection process of the results of these actions, it was possible for the teacher to evaluate the process of implementing an active learning methodology. The main characteristic of the PBL application is the need to change the teacher's mentality and behavior, since it has to create an environment where the learning process becomes student-centered, and the teacher needs to instigate it to learn, demonstrating the importance and developing their autonomy. The use of methodologies that bring student closer to the situations that he will encounter in his professional life guarantees a greater engagement of them in the classes. The study presented here evidenced the importance of the inclusion of active methodologies in classrooms, since the ability of students to learn is better developed. However, since there is no ready formula for applying these methodologies, the understanding, choice and

Educação, Psicologia e Interfaces, Volume 2, Número 2, p. 43-56, Maio/Agosto, 2018.

ISSN: 2594-5343. DOI: https://doi.org/10.37444/issn-2594-5343.v2i2.63 
application of active learning methodologies require a great dedication of the teacher throughout the teaching process.

KEYWORDS: Education. Active Learning. Psychology.

\section{INTRODUÇÃO}

Há um provérbio muito interessante sobre mudanças, que diz que "pedras que rolam não criam musgo", ou seja, quem se move não fica sujeito a estagnações, podendo estar apto à novas ideias. As mudanças na sociedade reverberam também dentro da universidade, onde atualmente a principal alteração se dá através da aplicação das metodologias de aprendizagem ativa nas salas de aula. Com isso, pretende-se trazer o estudante para baixo dos holofotes do conhecimento, sendo ele o principal responsável na construção do seu processo de aprendizagem, transformando o professor em um facilitador do processo (MELO; SANT’ANA, 2012).

Essa mudança altera bastante o status quo na sala de aula, pois tanto os estudantes como docentes precisam alterar sua forma de agir, pois ambos estavam acostumados à uma situação de o professor, sendo o dono do conhecimento, repassava todo o conteúdo de referido assunto e cabia ao estudante absorver tudo isso. O que havia é apenas uma via de mão única, com a informação sendo transmitida apenas do professor para o estudante. A inclusão das metodologias de aprendizagem ativa não interrompeu essa via. O que se obteve com isso foi a ampliação da mesma com a criação de uma outra faixa que permite com que o conhecimento possa ir e vir do docente ao estudante, e vice-versa (AMBROSE et al., 2010). E essa nova via criada na estrada do conhecimento traz várias perguntas ao docente, sendo a principal delas como entender o funcionamento de uma metodologia ativa de aprendizagem e ao mesmo tempo aplicá-la em sala de aula?

Quando se ensina uma disciplina através de metodologias ativas, é ideal que o professor considere trabalhar em uma aprendizagem mais profunda, onde os reflexos do ensino serão percebidos não apenas durante a disciplina, e sim isso irá transcorrer durante todo o curso de graduação e, principalmente, durante o exercício da profissão. O professor deve analisar se a função dele será a de informar, ou seja, ser um mero transmissor da informação ou ele pretende atuar como uma espécie de treinador, capacitando os 
estudantes para o desenvolvimento de pensamentos de ordem superior e de habilidades profissionais (LOYENS et al., 2015).

A literatura apresenta uma variedade de metodologias de ensino, cada uma com suas particularidades, e o desafio do docente é encontrar aquela, ou aquelas, que melhor se encaixam no que ele acredita ser o melhor método para que seus estudantes possam compreender o assunto. Dessa forma, a Pontifícia Universidade Católica do Paraná (PUCPR) capacitou seu corpo docente para que os mesmos se sentissem encorajados a aplicar metodologias ativa de aprendizagem em alguma de suas disciplinas.

O desafio proposto, descrito nesse artigo foi o de desenvolver técnicas de aprendizagem ativa na disciplina de Psicofarmacologia para estudantes do $5^{\circ}$ período do curso de Psicologia da PUC-PR. A grande pergunta a ser respondida com o desenvolvimento de uma técnica de metodologia ativa de aprendizagem era como abordar um tema bastante específico da área de Farmácia dentro de uma disciplina do curso de Psicologia?

Com essa situação que a referida disciplina apresenta, optou-se pela aplicação da metodologia de aprendizagem ativa denominada Aprendizagem Baseada em Problemas (PBL - Problem Based Learning), que tem como característica apresentar um problema que não apresenta uma solução pré-definida. Nessa metodologia não se foca muito na resolução do problema em si, mas sim no empenho na identificação do problema, a fim de, analisando as evidências apresentadas e utilizando argumentos lógicos, avaliar e propor soluções para o caso em questão. Tais ações desafiam os estudantes a raciocinar, argumentar e refletir para, por meio de análises e discussões individual e coletiva, chegar à uma decisão, desenvolvendo o raciocínio crítico e argumentativo dos estudantes, habilidades com alto poder cognitivo e social (DUCH, GROH; ALLEN, 2001; WALKER et al., 2015).

A apresentação de problemas através da metodologia de PBL visa aproximar cada vez mais o estudante das situações que serão vivenciadas no mundo real, ou seja, as situações a serem solucionadas são autênticas e complexas e a solução desconhecida, pois o que se pretende avaliar não é tanto o resultado, mas sim o caminho que o estudante percorreu para chegar à sua resposta. Através desse processo, a capacidade de aprender passa a ser atingida de forma mais eficaz (BOUD; FELETTI, 1999). A A implementação do PBL envolve a narrativa de problemas profissionais, para focar e motivar os estudantes, através da atuação em equipes, tendo o professor como facilitador 
do processo a fim de estimular o debate através de questionamentos, apresenta como principais características a necessidade, por parte do estudante, de uma formação autodidata orientada aliada à uma forte habilidade de trabalho em equipe (WALKER et al., 2015; RIBEIRO, 2011). O objetivo deste artigo foi relatar a experiência didática das dificuldades encontradas pelo professor no desenvolvimento das metodologias ativas de aprendizagem de PBL na disciplina de Psicofarmacologia do curso e Psicologia.

\section{MATERIAL E MÉTODO}

Para o desenvolvmento do processo de PBL, a primeira ação foi a divisão da turma em equipes, contendo entre 4 e 5 componentes, sendo que a escolha dos membros das equipes foi uma atribuição do professor, e não dos estudantes. Tal ação visou tornar os grupos o mais heterogêneo possível, pois um dos objetivos do PBL é estimular o debate entre os grupos. Durante o semestre foram apresentados à turma 16 diferentes problemas, distribuídos em módulos relativos ao tratamento farmacológico de psicopatologias (depressão, esquizofrenia, mania e bipolaridade) e para cada módulo, haviam casos diferentes para cada equipe, onde a diferença residia nas características do paciente. Com isso pretendeu-se evitar a troca de informação entre as equipes.

A proposta era que cada equipe fosse capaz de propor um tratamento farmacológico, adequado, seguro e eficaz, para o seu caso clínico. Como a disciplina compreendia encontros semanais de apenas 2 horas/aula, para que as atividades fossem realizadas na sua plenitude, as mesmas foram divididas em duas partes. A primeira parte, realizada apenas pelos discentes, de forma individual e fora da sala de aula, visava desenvolver a autonomia dos estudantes. Na segunda parte, realizada dentro da sala de aula, ocorria a discussão entre os membros dos grupos para a construção de uma resposta única ao problema proposto. Nessa etapa o professor atuou como facilitador do processo, circulando entre os grupos para verificar o progresso, questionar os motivos das concordâncias e discordâncias durante o entendimento da situação apresentada, além de solucionar dúvidas existentes referente ao processo de PBL.

A última etapa, realizada em grupo e dentro da sala de aula, tinha como objetivo desenvolver ainda mais o senso crítico dos estudantes, onde cada grupo recebeu tanto a descrição como a resolução do problema de outra equipe, os estudantes deveriam, através da discussão nos grupos, a partir do seu conhecimento obtido durante o seu processo de 
Aplicação de metodologia problem-based learning a partir do ponto de vista do professor

aprendizagem, analisar se a resposta fornecida pelo outro grupo para o problema apresentava coerência. Para tanto, foi fornecida aos grupos uma rubrica contendo os itens a serem avaliados e a pontuação a ser conferida.

\section{RESULTADOS E DISCUSSÃO}

Antes de iniciar o processo de ensino utilizando metodologias ativa, o professor deve ser capaz de definir três questões básicas que o irão guiar na tanto na montagem como no desenvolvimento da disciplina, o que Biggs (1996) denomina como alinhamento construtivo. A primeira é definir quais são os resultados de aprendizagem esperado para essa disciplina, ou seja, o que os estudantes devem saber e serem capazes de fazer ao final dessa disciplina. Em seguida, definem-se quais serão as atividades de ensino e aprendizagem, ou seja, descobrir quais atividades são apropriadas para os estudantes atingirem a aprendizagem desejada. A etapa final é a avaliação dos resultados de aprendizagem, caracterizado por saber como os estudantes demonstram que eles atingiram o nível desejado de aprendizagem. A construção desse alinhamento permite ao professor verificar quais das estratégias de aprendizagem ele irá utilizar (BIGGS, 1999).

Tendo isso em mente, optou-se por não realizar nenhuma aula expositiva tradicional para explicar conceitos de farmacologia. Como recurso para isso foi solicitado que, a medida que termos técnicos relativos à farmacologia fossem surgindo na busca de informações de literatura, cada estudante deveria montar um glossário com esses termos, para ser utilizado durante as aulas. Essa ação visava desenvolver nos estudantes a capacidade de perceber o que deveria ser estudado, dando a eles uma maior autonomia e permitindo ao professor atuar como facilitador do processo de aprendizagem.

Em virtude matriz curricular de Psicologia não apresentar uma base forte relacionado à farmacologia, era esperado que houvesse muitas dúvidas referentes ao assunto, pois termos abordados como farmacocinética, caracterizado como o percurso que o medicamento percorre dentro do organismo, ou farmacodinâmica, que é o modo como o medicamento atua dentro do corpo (GOODMAN; GILMAN, 2010) não são de conhecimento de estudantes e profissionais que não estejam envolvidos diretamente com medicamentos, como farmacêuticos, médicos e enfermeiros. Porém, o psicólogo atua diretamente com pacientes em tratamento farmacológico, e o conhecimento de como os medicamentos para psicopatologias atuam é essencial no auxílio do tratamento psicológico. 
Nessa etapa esperava-se uma grande resistência e dificuldade por parte dos estudantes, pois como dito anteriormente, o PBL contempla uma formação autodidata bastante significativa. Enquanto em uma abordagem mais tradicional de ensino o professor ensina o que precisa ser ensinado, o estudante memoriza o assunto e depois é apresentado um problema que ilustra o ensinamento, a linha mestra de uma aprendizagem através de PBL inverte essa lógica. Após a apresentação do problema, o estudante precisa ser capaz de identificar o que precisa ser aprendido e, a partir daí aprender e aplicar na resolução do problema (BOUD; FELETTI, 1999).

Assim, a opção pela não realização de aulas expositivas sobre conceitos demonstrou ser acertada, pois os estudantes foram instigados a descobrir o que precisavam aprender para resolver os problemas. Como resultado disso, os glossários apresentavam conteúdos bastante diversos e isso acabou gerando discussões dentro dos grupos, o que auxiliou bastante no entendimento do tema, pois a troca de informação entre os componentes permitiu melhorar a compreensão de termos. Mesmo que muito dos tópicos inseridos nos glossários fossem os mesmos, o entendimento era diferente entre os membros das equipes, e assim, a discussão dentro dos grupos, muitas vezes auxiliou na compreensão dos temas. Entretanto, como o assunto em questão, farmacologia, não é de um amplo conhecimento dos estudantes de Psicologia, o professor teve que atuar tanto dentro dos grupos como também de uma forma mais ampla, parando as discussões e realizando uma pequena aula expositiva sobre os tópicos.

A atividade inicial visava aos estudantes atingirem os níveis iniciais da Taxonomia de Bloom, ou seja, deviam ser capazes de recordar e compreender as diferentes psicopatologias, para a partir disso, conseguirem atingir o terceiro nível, que era o de aplicar os conhecimentos para usar a informação em novas situações (ANDERSON et al., 2001). Ao identificar qual patologia era abordada no problema, os membros das equipes deveriam elaborar uma lista individual contendo os medicamentos utilizados para o tratamento da referida enfermidade. Após essa atividade, realizada fora da sala de aula, as equipes se reuniam e discutiam, durante as aulas e com a supervisão do docente, quais dos medicamentos contidos nas listas individuais eram os mais adequados para o tratamento do caso clínico exposto.

Nas fases seguintes do projeto o objetivo era que os discentes fossem escalonando dentro dos níveis de pensamento da Taxonomia de Bloom, ou seja, serem capazes de, 
através da realização de conexões entre as ideias dos membros, analisarem quais as melhores opções para a resolução do problema. Atingindo esse nível, era possível os estudantes alcançarem os níveis mais altos de pensamento superior da referida taxonomia, caracterizados pela capacidade de avaliar suas conclusões, justificando a decisão e por fim serem capazes de criar uma resposta única, através da produção da resolução do problema.

A maior dificuldade percebida pelo docente ao realizar essa ação foi a de saber tanto quando atuar e como atuar, pois a prática de PBL envolve tanto a autonomia de aprendizagem como também aprendizagem colaborativa. Como o docente atua mais como facilitador do processo do que propriamente como professor, ele deve instigar a discussão entre os grupos, a fim de que os membros possam trocar ideias e chegar a respostas. Porém, é primordial que o professor saiba perceber quando a compreensão dos assuntos não está sendo totalmente obtida, ou quando o caminho que a equipe está seguindo levará a respostas erradas, e a partir daí intervir, seja questionando os motivos que levaram o grupo à essas direções, e a partir desses questionamentos fazer o grupo perceber onde houve equívoco, ou seja também, em casos específicos, explicar o assunto não compreendido. E essa foi uma dificuldade percebida pelo professor na realização dessa disciplina, ou seja, saber quando e de que maneira agir para auxiliar a compreensão dos assuntos sem frear o processo de autonomia de aprendizagem.

Outro detalhe que ficou evidenciado nessas duas fases foi a importância da capacidade de aprender por si mesmo. Durante as discussões internas, percebeu-se que os estudantes que conseguiram identificar o que deveria ser aprendido para resolver o problema proposto tiveram maior capacidade de argumentação para expor e convencer os demais do seu ponto de vista. E com isso, os demais perceberam a necessidade de um maior engajamento para que o processo de aprendizagem se fortalecesse. Conforme Yew e Goh (2016) a filosofia do PBL é que a aprendizagem pode ser considerada uma atividade construtiva, auto direcionada e colaborativa.

A efetividade do PBL apresenta resultados diferentes, dependendo do ponto de vista. Estudos demonstram que, a curto prazo, a capacidade de aprendizagem através de PBL é bastante similar à obtida por uma metodologia tradicional. Porém, a retenção do conhecimento através do PBL, é muito maior quando se analisa o processo à longo prazo, demonstrando a efetividade da metodologia para a vida profissional o estudante (DOCHY et al., 2003; POURSHANAZARI et al., 2013; CAPON; KUHN, 2004). 
$\mathrm{Na}$ etapa final, cada equipe deveria entregar uma resposta única visando resolver o problema proposto, ou seja, deveriam apresentar um tratamento farmacológico para o caso clínico apresentado, com todas as justificativas técnicas que corroborassem para essa resposta. Foi possível perceber a evolução do entendimento, e por consequência, das resoluções propostas aos problemas apresentados, a medida que os casos iam sendo resolvidos. De uma forma bastante surpreendente e positiva para o professor, a primeira resolução dos problemas apresentou um bom embasamento teórico a fim de justificar as decisões tomadas. A medida que novos problemas eram apresentados, os conceitos básicos de farmacologia e a compreensão de todos os aspectos que envolvem a resolução de um problema iam ficando cada vez mais bem compreendidos, e isso resultou em uma melhora significativa nas respostas dos problemas. Aliado a isso, a apresentação de problemas que os estudantes irão encontrar durante sua vida profissional também foi fundamental para a aceitação por parte da turma, desse tipo de metodologia.

Outro detalhe percebido na aplicação dessa metodologia foi referente à montagem dos grupos. Na aplicação de uma metodologia ativa, a montagem dos grupos é fundamental, pois como o PBL baseia-se em aprendizagem colaborativa, equipes malformadas podem causar impacto negativo na aprendizagem a medida que a compreensão e discussão dos assuntos não seja bem realizada. Para essa atividade, a divisão das equipes foi realizada através da aplicação de um questionário, onde além de responder questões como cidade de residência, se trabalha, faz estágio apenas estuda, os estudantes responderam um pequeno teste neurolinguístico a fim de descobrir se sua capacidade de aprendizagem era mais visual, auditiva ou cinestésica. Dessa forma, os grupos foram montados de forma a ter componentes com características diferentes.

Mesmo tal atividade sendo atribuída ao professor e as equipes formadas apresentaram heterogeneidade em relação às respostas do questionário, alguns grupos acabaram sendo compostos por estudantes com maior capacidade de autoaprendizagem e/ou cognitiva, e isso foi perceptível nas respostas às questões propostas. Por mais que houvesse uma harmonia nas resoluções, as equipes compostas por estudantes que já possuíam ou desenvolveram mais rapidamente as habilidades citadas apresentavam respostas com maior embasamento teórico em relação aos demais.

Isso demonstrou a dificuldade da divisão da turma em grupos, pois ao romper um velho paradigma, o dos próprios estudantes formarem suas equipes através da afinidade, 
Aplicação de metodologia problem-based learning a partir do ponto de vista do professor

pretendeu-se criar equipes heterogêneas internamente, porém que apresentasse uma homogeneidade quando avaliado de fora. E nem sempre esse objetivo é atingido, pois seja qual for o critério, sorteio ou através de questionário, grupos de maior e de menor capacidade cognitiva acabam sendo criados. A melhor maneira para que grupos com alto grau de heterogeneidade sejam criados é quando o professor conhece bem as características dos indivíduos que compõem a turma, e assim consegue fazer uma distribuição manual dos discentes de forma que a equipe seja composta por alunos com perfis diferentes.

Como forma de avaliar os estudantes, como o PBL visa não apenas o resultado, e sim todo o processo, optou-se por realizar, juntamente com uma avaliação somativa, onde cada etapa (individual e coletiva) gerava uma nota, a avaliação formativa, onde o professor avaliava como os estudantes evoluíam a sua capacidade de aprendizagem durante a resolução do problema proposto. A utilização desse tipo de avaliação da turma foi fundamental, pois não apenas permitiu ao professor acompanhar o desenvolvimento do processo de aprendizagem dos estudantes, e sim possibilitou ao estudante verificar quais eram seus pontos fortes e fracos na sua aprendizagem e saber capitalizar e melhorar tais pontos.

A implementação de um PBL visa inserir o estudante em situações autênticas do mundo real. Geralmente, dentro do mundo acadêmico, na forma tradicional de ensino, quando se apresentam problemas para resolução na sala de aula, as situações criadas quase sempre não são autênticas e de certo ponto fragmentadas e, de forma bastante comum, a solução para resolvê-lo é conhecida, pois isso facilita o processo de avaliação, já que a intenção, na maioria das vezes, é o professor avaliar se o estudante obteve a resposta considerada correta. Em muitos casos, o estudante consegue chegar à essa resposta final, porém o processo de aprendizagem não é totalmente atingido (BOUD; FELETTI, 1999).

A etapa final para a resolução dos problemas propostos contemplava a avaliação por pares, onde a descrição do problema e a resolução proposta para o mesmo de cada grupo foi distribuído aos demais grupos. Para essa tarefa, a distribuição das tarefas também ficou a cargo do professor, pois pretendeu-se a realização de uma análise cega, ou seja, cada equipe não saberia qual grupo estavam avaliando, e com isso o foco da análise por pares ficou centrada no conteúdo descrito e não em quem descreveu. A utilização dessa atividade visava desenvolver a capacidade de análise e avaliação dos 
estudantes, na medida que eles deveriam fazer uma análise crítica da resolução proposta por um outro grupo e avaliar se a resposta formulada apresentava concordância tanto com a literatura como com os conhecimentos desenvolvidos pelos mesmos na realização da atividade.

Como a tarefa de avaliar e corrigir um trabalho não é uma tarefa simples, o professor desenvolveu e entregou aos estudantes uma rubrica do tipo analítica. Essa rubrica é uma ferramenta onde se listam todas as expectativas de uma atividade a ser realizada através da divisão em etapas e proporcionam a descrição dos níveis de desempenho, como por exemplo, aceitável ou não. A construção de uma rubrica eficaz, que seja de fácil compreensão e aplicação envolve os seguintes passos: (i) identificar a tarefa a ser avaliada; (ii) listar os componentes a serem avaliados; (iii) determinar a ponderação dos componentes; (iv) listar os critérios de avaliação; (v) escala de apreciação dos níveis de aquisição; (vi) descrição dos níveis de aquisição e (vii) comentários. A rubrica de avaliação foi utilizada tanto para a avaliação por pares por parte dos estudantes como também pelo professor, a fim de garantir que os parâmetros avaliados fossem os mesmos aceitável (STEVENS; LEVI, 2005)

O processo final de avaliação através das rubricas teve ponderações distintas. Em virtude de a capacidade de avaliação crítica ser uma habilidade a ser desenvolvida nos estudantes, a nota atribuída por um grupo para a resolução do problema de outra equipe teve pequeno percentual na composição da nota final, cabendo à avaliação realizada pelo professor ter uma ponderação maior na nota. Entretanto, como o objetivo dessa atividade de avaliação por pares era atribuir nos estudantes uma responsabilidade de avaliação, através da construção de um senso crítico mais desenvolvido, foi atribuído um percentual de nota referente à capacidade de análise crítica da resolução de um problema. Isso garantiu que a avaliação por pares fosse realizada com bastante critério por parte dos estudantes.

A avaliação da resolução do primeiro problema apresentado forneceu bons resultados pelo ponto de vista do entendimento dos estudantes da metodologia PBL. Pelo fato de os discentes estarem acostumados à uma cultura tradicional de ensino, com o professor atuando como detentor do conhecimento e os alunos como receptores da informação, ao inverter essa lógica, a turma foi retirada da sua zona de conforto, logicamente com diferenças na velocidade com que cada estudante saía dessa zona. 
Aplicação de metodologia problem-based learning a partir do ponto de vista do professor

Foi possível perceber, na resolução do primeiro problema, claramente a construção do processo de aprendizagem ser desenvolvido. A necessidade de os estudantes terem que retomar conceitos de disciplinas lecionadas em semestres anteriores, além de terem que aprender assuntos novos sozinhos, apenas com a orientação do professor, fez com que a turma fosse entendendo não só o funcionamento de uma metodologia ativa de aprendizagem, mas, principalmente, sendo capazes de aprender tanto um novo assunto como também aprender a aprender. Essa construção da aprendizagem ficou mais perceptível quando se comparou o processo de resolução do primeiro problema apresentado com os demais. A capacidade de atingir os níveis mais altos da escala da Taxonomia de Bloom foi se tornando cada vez mais rápida e melhor, de forma que as resoluções dos problemas propostos foram ficando cada vez melhor, com maiores argumentações teóricas que sustentasse a opinião da equipe.

Porém, a maior surpresa na aplicação do PBL foi o resultado das avaliações por pares. De maneira geral, estudantes não estão acostumados e atuarem como avaliadores, principalmente de colegas. Muito disso decorre do fato de o processo tradicional de aprendizagem visar mais a transmissão de conteúdo do professor para o aluno, que retém esse conhecimento e o uso na hora de provas. Ser capaz de avaliar, com critério, qualquer trabalho, seja ele acadêmico ou profissional, exige em um elevado conhecimento do assunto abordado, não só no que se refere aos acertos, mas principalmente nas justificativas que vem a corroborar com as respostas. Também é fundamental que o avaliador, nesse caso o estudante, seja capaz de perceber erros e/ou equívocos referentes à compreensão do tema, que podem direcionar à uma resposta que não seja a mais adequada para o problema proposto.

Ao comparar as notas atribuídas pelos estudantes na avaliação por pares com a atribuída pelo professor, percebeu-se que havia uma proximidade entre as mesmas, demonstrando que houve o entendimento, por parte da turma, de como realizar uma avaliação. Entretanto, é importante ressaltar que a inclusão de uma rubrica de avaliação para um direcionamento de quais itens a serem avaliados, e como deveriam ser avaliados, facilitou a realização da avaliação por pares. Em uma avaliação de qualidade, o instrumento de medida tem validade apenas se é possível medir o que deve ser medido e tem fiabilidade se fornece resultados semelhantes, independentemente de quem, quando e onde seja feita a avaliação (HALADYNA; RODRIGUEZ, 2013). 
O entendimento do funcionamento, e por consequência da aplicação de uma metodologia ativa de aprendizagem é algo que não se constrói rapidamente. Envolve um aprendizado constante e uma grande capacidade de mudança de mentalidade, não só dos acadêmicos, mas principalmente do professor. A experiência obtida através da aplicação do PBL demonstrou ser altamente positiva para o professor, pois foi possível alterar a aprendizagem, de forma que os estudantes pudessem entenderem a disciplina para atingirem os resultados de aprendizagem desejados e não apenas decorarem conceitos.

\section{CONSIDERAÇÕES FINAIS}

A partir dessa experiência, confirmou-se que em metodologias ativas de fato não existe aula completamente pronta, podendo ser replicada identicamente em outras turmas, pois cada turma fará com que ações as atividades sejam adaptadas para atender os resultados de aprendizagem.

A fim de que uma metodologia ativa seja aceita e entendida pelos estudantes, deve haver uma relação muito direta e próxima do que os mesmos vivenciarão no mundo profissional. Como as metodologias mais tradicionais, na maior parte dos casos, se apoiam muito em conceitos teóricos, que são fundamentais para moldar o conhecimento do assunto, metodologias ativas apresentam, seja ela qual for, apresenta o mundo real e prático, fazendo com que o estudante saia da sala e adentre na sua profissão ainda durante uma aula. A utilização de metodologias que aproximem o estudante das situações que ele encontrará na sua vida profissional garante um maior engajamento dos mesmos nas aulas, pois dá a eles um certo domínio sobre como a aula irá transcorrer e como resolver problemas, através da discussão entre os membros da equipe.

\section{AGRADECIMENTOS}

O autor agradece o Suporte financeiro do FINEP para a implementação da prática e realização da pesquisa.

\section{REFERÊNCIAS BIBLIOGRÁFICAS}

AMBROSE, S.; BRIDGES, M.W.; DIPIETRO, M.; LOVETT, M.C.; NORMAN, M.K. How learning works: Seven Research-Based Principles for Smart Teaching. San Francisco: John Wiley \& Sons, 336 p., 2010. 
ANDERSON, L. A taxonomy for learning, teaching and assessing: a revision of Bloom`s taxonomy of educational objectives. New York: Longman, 2001.

BIGGS, J. Enhancing teaching through constructive alignment. Higher Education, v. 32 (3), pp. $347-364,1996$.

BIGGS, J. What the students does: Teaching for enhanced learning. Higher Education Research \& Development, v 18 (1), pp. 57 - 75, 1999.

BOUD, D.; FELETTI, G. (Ed). The Challenge of Problem Based Learning. $2^{\text {nd }}$ ed. London: Kogan Page, 1999;

CAPON, N.; KUHN, D. What's so good about problem-based learning? Cognit Instr, 22, (1), p. 61-79, 2004.

DOCHY, F.; SEGERS, M.; VAN DEN BOSSCHE, P.; GIJBELS, D. Effects of problem-based learning: a meta-analysis. Learn Instr, 13 (5), p. 533-568, 2003.

DUCH, B.J.; GROH, S.; ALLEN, D.E. (Ed). The Power of Problem-Based Learning. Virginia: Stylus Publishing, 2001.

HALADYNA, T. M. E RODRIGUEZ, M. C. Developing and validating test items. New York, NY: Routledge, 2013.

LOYENS, S.M.M.; JONES, S.H.; MIKKERS, J.; VAN GOG, T. Problem-based learning as a facilitator of conceptual change. Learn Instr, 38, p. 34-42, 2015.

MELO, B.C.; SANT’ANA, G. A prática da Metodologia Ativa: compreensão dos discentes enquanto autores do processo ensino-aprendizagem. Com. Ciências Saúde. 23 (4), 327-339, 2012.

POURSHANAZARI, A.A; ROOHBAKHSH, A.; KHAZAEI, M.; TAJADINI, H. Comparing the long-term retention of a physiology course for medical students with the traditional and problem-based learning. Adv Health Sci Educ, 18 (1), p. 91-97, 2013.

RIBEIRO, Luis Roberto. The Pros and Cons of Problem-Based Learning from the Teacher's Standpoint. Journal of University Teaching \& Learning Practice. 8, (1) Article 4, 2011

STEVENS, D.D.; LEVI, A J. Introduction to rubrics. An Assessment Tool to Save Granding Time, Convey Effective Feedback and Promote Student Learning. Sterling, VA: Stylus, 2005

VERNON, D T; BLAKE, R L. Does problem-based learning work? A meta-analysis of evaluative research. Academic Medicine, 68, (7), p. 550-63, 1993.

WALKER, A.; LEARY, H. HMELO-SILVER, C. E.; ERTMET, P.A. (Ed). Essential readings in Problem-Based Learning. West Lafayette: Purdue University Press, 2015. 
YEW, EHJ; GOH, K. Problem-Based Learning: An Overview of its Process and Impact on Learning. Health Professions Education. 2, (2), p.75-79, 2016.

\section{Credenciais do autor}

SAUSEN, Tiago Rafael. Atualmente é professor dos cursos de Biomedicina, Nutrição e Fisioterapia na Univel (União Educacional de Cascavel) Mestrado em Ciências Farmacêuticas pela Universidade Federal do Rio Grande do Sul (UFRGS Farmacêutico pela Universidade Regional do Noroeste do Estado do Rio Grande do Sul (UNIJUÍ)).

Endereço para correspondência: Tiago Rafael Sausen, cursos de Biomedicina, Nutrição e Fisioterapia na Univel, Av. Tito Muffato, 2317 - Santa Cruz, Cascavel - PR, 85806-080. E-Mail: tiago.sausen@ pucpr.br

Como citar este artigo (Formato ABNT): SAUSEN, Tiago Rafael. Aplicação de metodologia problem-based learning a partir do ponto de vista do professor. Educação, Psicologia e Interfaces, v.2, n.2, p. 43-56, 2018. DOI: https://doi.org/10.37444/issn2594-5343.v2i2.63

Recebido: 04/02/2018.

Aceito: 26/06/2018. 